Citation: Lincoln NB, Radford KA, International Journal of Therapy and

Rehabilitation, June 2014, Vol 21, No 6

\title{
A shortened version of the Dementia Drivers' Screening Assessment
}

\author{
Nadina Lincoln ${ }^{1}$ and Kate Radford ${ }^{1}$ \\ ${ }^{1}$ Division of Rehabilitation and Ageing, University of Nottingham, Nottingham, UK \\ Email: Nadina Lincoln - nadina.lincoln@nottingham.ac.uk, Kate Radford - kate.radford@nottingham.ac.uk
}

\begin{abstract}
Introduction: Cognitive tests are used to inform recommendations about the safety of people with dementia to continue driving. The Dementia Drivers' Screening Assessment (DDSA) is a neuropsychological battery designed to assist in this process. However, it is lengthy to administer and requires materials from various test batteries.
\end{abstract}

Aims: The primary aim of this study was to develop a shortened version of the DDSA for individuals with dementia.

Methods: Data on participants with dementia from two studies were analysed. These participants were all drivers with dementia who were identified by community mental health teams and psychiatrists. Each participant was assessed on the DDSA and also assessed on-road by an 'approved driving instructor' using the Nottingham Neurological Driving Assessment.

Results: This study analysed 102 participants, who had a mean age of 74.0 (SD=7.7) years and of whom 80 (78\%) were men. Twenty three drivers were judged to be unsafe and 79 safe. The agreement between the short version and on-road assessment was $79 \%$. The assessment was better at detecting safe drivers than unsafe drivers.

Conclusion: The findings suggested that the shortened DDSA is suitable for participants who are unable or do not wish to undergo lengthier assessment.

People with dementia in the UK must notify the Driver and Vehicle Licencing Agency (DVLA) of their diagnosis and their licence is then regularly reviewed. The DVLA asks the medical person responsible for their care, usually a general practitioner or psychiatrist, for further information. This includes information about their cognitive abilities in order to help decide whether the person has the cognitive skills needed to continue driving. Health professionals, such as occupational therapists and psychologists, are frequently asked for their opinions on patients' abilities in order to inform the recommendation to the DVLA.

Clinicians sometimes use cognitive tests to inform these recommendations, but not always. Wilson and Pinner (2013) pointed out that the challenge is assessing the level of risk of a driver with dementia and determining the level of risk that is unacceptable. They highlighted that the procedures used in clinical practice are very subjective. However, cognitive tests 
have been developed to assist in this process, and to provide more standardised procedures.

Other countries use similar processes (Seiler et al, 2012), but there is a lot of variation in the methods used and discrepancies between countries have been highlighted in the Alcove Report (Nys and Raeymaekers, 2013) and by Alzheimer Europe (2014). In some countries, e.g. Belgium and Australia, on-road assessment is common, but even then there is still the decision to be made about when to conduct the on-road assessment. In other countries, there are no routine on-road assessments and the decision to withdraw a licence is made solely on the basis of medical opinion. Cognitive tests are used in some countries e.g. Finland, but not routinely in most. This variation in procedures has highlighted the need for better validated methods.

An on-road assessment is usually considered to be the gold standard measure of driving ability (Reger et al, 2004). Therefore, in countries where not everyone is tested on the road, it is important that the cognitive tests used have been validated in relation to on-road driving.

Research has shown an association between performance on cognitive tests and the ability to drive on the road in people with dementia (Reger et al, 2004, Molnar et al, 2006). A systematic review of studies examining the relation between neuropsychological function and driving ability in people with dementia identified 27 studies, 12 of which used on-road driving to assess driving ability (Reger et al, 2004). Reger et al (2004) grouped the neuropsychological tests according to the cognitive domains assessed and reported that the effect sizes were significant but small for the relation between on-road driving and all neuropsychological tests in patients with dementia; however, effect sizes were greatest for measures of visuospatial skills.

Molnar et al (2006) conducted a similar review but instead examined each test separately. They identified 16 studies that examined the relation between cognitive tests and driving ability, but only six used on-road driving as the measure of driving ability. Molnar et al (2006) found marked inconsistencies between studies, with tests showing positive associations with driving in some studies but not in others. They also identified the problem that very few studies provided cut-off scores for tests that could be used to make clinical decisions in individual patients.

Cognitive tests serve two purposes relating to driving for people with dementia. One is to screen people to identify who needs on-road assessment, and the second is that they can form part of a comprehensive driver evaluation. Some decisions can be made without the need for on-road assessment. People who have very mild cognitive impairment will be safe to drive, while those with very severe cognitive impairment will be unsafe. Cognitive screening aims to identify those with borderline cognitive abilities, so that these people are referred for specialist on-road assessment. This means that not every driver with dementia 
will be assessed at a specialist driving assessment centre. Cognitive assessment is an integral part of a comprehensive assessment of fitness to drive, which highlights potential areas of difficulty and informs the on-road assessment.

Some assessments have been developed for people with a range of aetiologies likely to affect driving ability. For example, the OT-DORA (Unsworth et al, 2012) was developed as an off-road driver assessment battery, which includes physical and sensory assessments in addition to cognitive test-ing. The OT-DORA is a comprehensive assess $\neg$ ment designed to inform occupational therapists about impairments that may affect the on-road assessment, and to identify clients who are not suitable for on-road assessment. However, the predictive validity for on-road driving has not yet been fully evaluated.

The Rookwood Driving Battery (RDB) (McKenna, 2009) is part of the overall evaluation of fitness to drive and is used in many of the UK's Forum of Accredited Mobility Centres as part of their safety to drive assessment procedures. It comprises 12 tests, which yield an overall score. A cut-off of $>10$ was recommended to identify those who were unsafe to drive (McKenna and Bell, 2007). This had a high positive predictive value as $85 \%$ of those who failed the RDB, were found to be unsafe to drive on the road. However, the ability to detect unsafe drivers was $54 \%$, meaning that of those who were unsafe to drive, only $54 \%$ were correctly identified by the RDB (sensitivity $54 \%$, specificity $66 \%$ ). It was suggested that for elderly people over 70 years, a cut-off of $>6$ points should be used. Using this, the positive predictive value was $78 \%$, the ability to detect unsafe drivers (sensitivity) was $66 \%$ and the ability to detect safe drivers (specificity) was $73 \%$. Assessments, such as the OT-DORA and $\mathrm{RDB}$, have been developed for people with a wide range of aetiologies, while others have been developed specifically for those in a particular diagnostic category, e.g. dementia.

The Dementia Drivers' Screening Assessment (DDSA) (Lincoln et al, 2010) was developed to identify the drivers with dementia who should be assessed on the road. The DDSA was validated with two samples of drivers with dementia who were attending memory clinics. Their performance on a battery of cognitive tests was compared with the results of the Nottingham Neurological Driving Assessment (Lincoln et al, 2012) an on-road assessment, which was conducted by an instructor blind to the cognitive test results. Discriminant function analysis was used to generate equations to classify patients as safe or unsafe to drive. The equations had good predictive validity for identifying drivers with dementia who were safe to continue driving (Lincoln et al, 2006) and this was supported in an independent validation (Lincoln et al, 2010).

Good screening tests need to have a high positive predictive value, i.e. those who fail the cognitive test are highly likely to be unsafe on the road. The positive predictive value of the DDSA for detecting unsafe drivers was $82 \%$ in the original sample. In the validation sample, the positive predictive value for unsafe drivers was lower (62\%). However, the negative predictive value, i.e. the number of people who passed the test who passed on the road, 
was high (96\%) in the initial sample (Lincoln et al, 2006), and $80 \%$ in the validation sample (Lincoln et al, 2010).

Tests also need to have high sensitivity, i.e. the test should correctly identify a high proportion of unsafe drivers. The sensitivity of the DDSA was $90 \%$ in the initial sample but only $44 \%$ in the validation sample (Lincoln et al, 2006; 2010). The DDSA was better able to detect safe drivers than unsafe drivers, as its specificity was $93 \%$ in the initial sample and $89 \%$ in the validation sample. On this basis, the test is recommended for deciding who is safe to drive, and those who fail the test should be referred for on-road assessment.

The materials necessary to administering the DDSA must be collated from a rage of cognitive tests used in clinical practice. This has proved expensive for clinical services that do not have the full range of tests available due to the cost of the tests. The DDSA is also lengthy and some people with dementia are reluctant to complete the entire battery. Therefore, this study aimed to develop a shortened version of the DDSA, the Nottingham Assessment for Drivers with Dementia (NADD) (Lincoln and Radford, 2012), which does not require tests from multiple sources. The tests were selected on the basis of availability and cost.

\section{METHODS}

Anonymised data were collated from previous research (Lincoln et al 2006; 2010). In these studies, psychiatrists and community mental health teams identified potential participants with any type of dementia.

Participants were included in the study if they: were diagnosed with dementia by their treating clinician; had no other medical diagnosis (e.g. stroke, multiple sclerosis) that could affect their driving; had driven a vehicle within the last 5 years; had a valid driving licence; lived within a 100 mile radius of the recruitment centre. All of the participants had previously consented to take part (Lincoln et al 2006; 2010).

Participants were assessed on the DDSA (Lincoln et al, 2010). The DDSA comprises the following tests: Mini Mental State Examination (Folstein et al, 2000) total score; the Stroke Drivers' Screening Assessment (SDSA) (Nouri and Lincoln, 1994) components, the Dot Cancellation shortened version (12 lines) time and errors, Square Matrices Directions and Road Sign Recognition; the Salford Objective Recognition Test (SORT) immediate and delayed recognition of words; the Stroop Colour-Word Test (Victoria version: Strauss et al, 2006) discrepancy between colour-word time and non-colour-words time; Visual Object and Space Perception (VOSP) (Warrington and James, 1991) incomplete letters; Behavioural Assessment of the Dysexecutive Syndrome (BADS) (Wilson et al, 1996) rule shift and key search profile scores; and the Adult Memory and Information Processing Battery (AMIPB) (Coughlan and Hollows, 1985) information processing A-adjusted score. Subtests from the SDSA, SORT, Stroop and AMIPB comprise the NADD. 
Participants were assessed on the road by an 'approved driving instructor', who was experienced in assessing people with dementia and was blind to the cognitive test results. The approved driving instructor met the participants and provided them with an overview of the driving assessment and answered any questions. The on-road assessments were conducted using the participants' own cars. They were assessed on the Nottingham Neurological Driving Assessment (Lincoln et al, 2012), which is a standardised on-road assessment comprising of 25 road manoeuvres. This assessment was conducted on a pre-planned route that included quiet roads, dual carriageways and busy town roads. Each manoeuvre was recorded as correct, a minor error (no effect on safety) or a major error (compromising safety). At the end of the drive, participants were graded as 'definitely unsafe', 'probably unsafe', 'probably safe' or 'definitely safe' to drive.

\section{RESULTS}

In the two studies, there were 102 drivers with dementia. The mean age of participants was 74.0 years $(S D=7.7$, range $52-88)$ and $80(78 \%)$ were men.

Discriminant function analysis was conducted to identify equations to classify safe and unsafe drivers on the NADD. The dependent variable was safety to drive as assessed on the road test. The independent variables were subtests from the SDSA, SORT, Stroop and AMIPB. These equa-tions were:

Pass equation:

(Dot cancellation time 12 lines * 0.058) + (Dot cancellation errors 12 lines * 0.092) + (Square Matrices Directions * 0.146) + (Road Sign Recognition * 0.470) + (SORT Words Immediate * 1.533) - (SORT Words Delayed * 0.326) + (Information Processing A-adjusted score * 0.156) $+($ Stroop * 0.012) -20.129 .

Fail equation:

(Dot Cancellation time 12 lines * 0.058) + (Dot Cancellation errors 12 lines * 0.128) + (Square Matrices Directions * 0. 138) + (Road Sign Recognition * 0.216) + (SORT Words Immediate * 1.209) - (SORT Words Delayed * 0.025) + (Information Processing A-adjusted score * 0.157) $+($ Stroop * 0.011) -20.304 .

Overall Score $=$ pass equation total - fail equation total

The overall conclusions from the NADD were cross-tabulated with the decisions about safety to drive from the on-road assessment. The results are shown in Table 1. There was $79 \%$ agreement between the classification of the NADD and the on-road assessment. The NADD was better at identifying safe drivers (specificity 94\%) than unsafe drivers (sensitivity $30 \%$ ). The positive predictive value was $58 \%$, so that just over half of those who failed the NADD were unsafe on the road, but the negative predictive value was $82 \%$, indicating that most people who passed the NADD were safe on the road. 
Receiver operating characteristic (ROC) curves were constructed to examine the ability of individual tests of the NADD to discriminate between safe and unsafe drivers. Road Sign Recognition and Square Matrices from the SDSA both produced a significant area under the curve (AUC). Road Sign Recognition $A \cup C=0.71,95 \% \mathrm{Cl}=0.59-0.84, \mathrm{P}=0.002$; Square Matrices $A \cup C=0.65,95 \% \mathrm{Cl}=0.52-0.79, \mathrm{P}=0.027$ (see Figure 1 ).

Using a cut-off score of $>6$ on Road Sign Recognition to indicate a safe driver had $36 \%$ accuracy overall, but $94 \%$ of those who achieved this score were found to be safe on the road. Similarly, a cut-off score of $>16$ on Square Matrices Directions had $46 \%$ accuracy overall, but $90 \%$ of those who achieved this score were found to be safe on the road. These cut-offs can be used to identify safe drivers. Those who score below either cut-off need to be assessed further using on-road assessments.

\section{Table 1. Classification of drivers with dementia on the basis of the NADD}

\begin{tabular}{|c|c|c|c|c|}
\hline \multicolumn{5}{|c|}{ On-road assessment } \\
\hline Result & & Fail & $\mathrm{Pa}$ & \\
\hline $\begin{array}{l}\text { Nottingham } \\
\text { Assessment } \\
\text { for Drivers }\end{array}$ & Fail & 7 & 5 & $\begin{array}{l}\text { Agreement }=79 \% \\
\text { Sensitivity for fail=30\% } \\
\text { Specificity }=94 \%\end{array}$ \\
\hline with Dementia & Pass & 16 & 74 & $\begin{array}{l}\text { Positive predictive value }=58 \% \\
\text { Negative predictive value }=82 \%\end{array}$ \\
\hline
\end{tabular}

Totals $(n)$

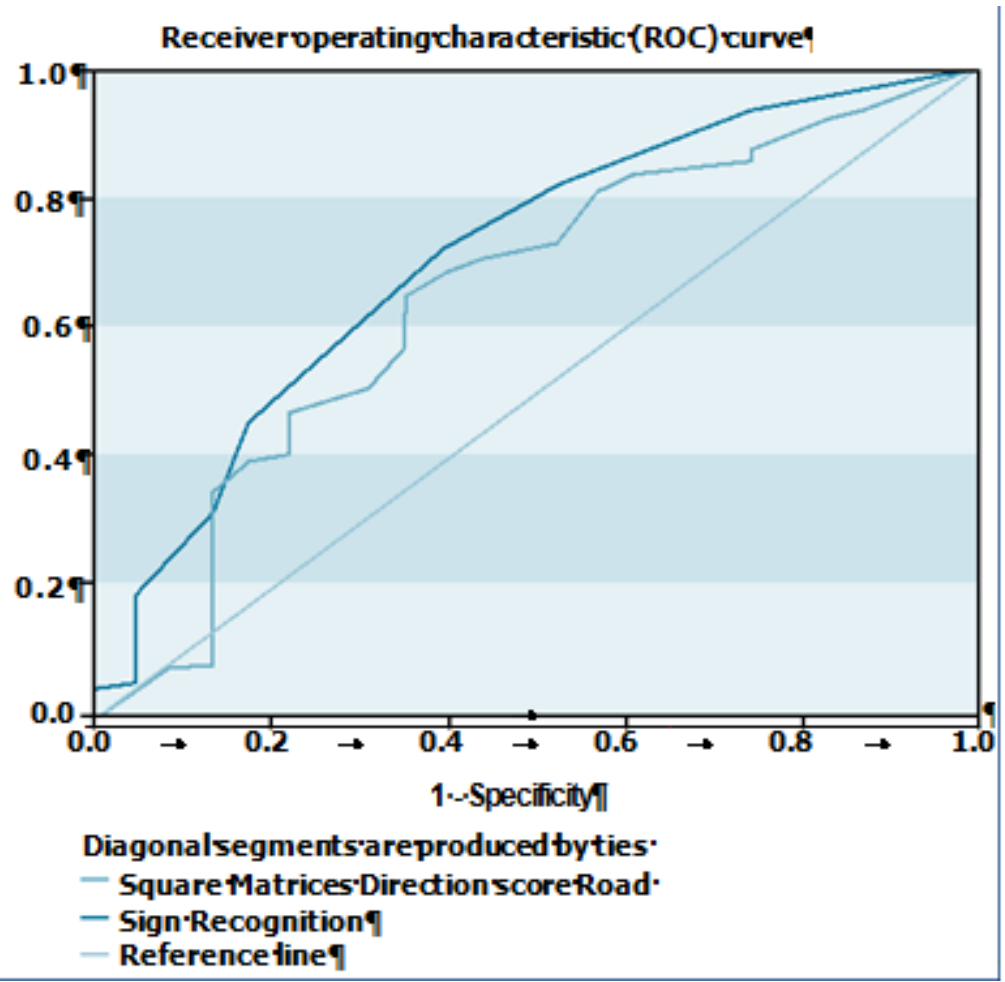

Fgure1. ROC curve to show discriminative ability of Road Sign Recognition and Square Matrices Directions tests 
The discriminant function analysis of the NADD showed that the shortened version of the test battery was able to classify $79 \%$ of drivers as safe or unsafe on the road. This is slightly lower than the accuracy of the full DDSA, which correctly classified 92\% of drivers in the initial sample (Lincoln et al, 2006) but slightly better than the $76 \%$ achieved in the validation sample (Lincoln et al, 2010). However, the NADD was only able to identify safe drivers and not those who were unsafe. In addition, equations developed for the NADD also need validation in an independent sample. What these findings suggest is that the NADD provides an acceptable alternative for people who are unable to tolerate the full DDSA, or for services that do not have the full range of test materials available, the shorter assessment may be used. The advantage of the NADD is that it is shorter than the DDSA, taking approximately two thirds of the time to administer, and is cheaper One criticism of both the DDSA and the NADD is that they use a complex scoring procedure. For this reason, individual tests were examined to assess their ability to differentiate between safe and unsafe drivers. Road Sign Recognition and Square Matrices Directions were both able to significantly differentiate between safe and unsafe drivers, but no cut-off point could be identified on either test with sufficient accuracy for making decisions about safety to drive. Drivers who scored more than 6 on the Road Sign Recognition or more than 16 on the Square Matrices Directions were highly likely to be safe on the road, but there were several drivers who scored below these cut-offs who were also safe.

\section{Limitations}

The limitations of the study are that this was a retrospective analysis of previously-collected data.

This meant that not all participants completed the same battery of tests. In addition, participants completed more tests than were included in the analysis. It may be that performance was worse on tests later in the battery due to tiredness and that people would have performed better on these tasks if the NADD had been used on its own. This means that a validation study is needed with an independent sample in which the predictions from the NADD are compared with on-road assessment, to confirm these findings. However, the complex scoring of the NADD remains a problem. The test has limitations in that it does not detect unsafe drivers, but it is acceptable for identifying those who are safe to continue driving and it is probably more accurate than using non-standardised or non-validated procedures, as occurs in many clinical services. Although the aim is to develop a test to detect unsafe drivers, the validation studies indicated that the NADD is more accurate at identifying safe drivers than unsafe drivers; those who are not predicted to be safe should be assessed on the road. The NADD has also been compared with the RDB (Vella and Lincoln, 2014) and found to show good agreement in the classification of safe and unsafe drivers when a cut-off score $>10$ is used to detect unsafe drivers on the RDB.

Although the two subtests, Road Sign Recognition and Square Matrices Directions, of the NADD were identified that significantly discriminated safe from unsafe drivers, their 
accuracy was not sufficient to allow them to be used on their own. However, the finding that two subtests of the SDSA were the best predictors of safety to drive is consistent with findings with stroke patients. Devos et al (2011) found that Road Sign Recognition and Square Matrices Compass were among the best individual predictors of safety to drive after stroke.

\section{CONCLUSIONS}

The NADD was able to identify drivers with dementia who were safe to drive. It is suitable for drivers with dementia who are not able to complete the full Dementia Drivers' Screening Assessment or when the full set of test materials is not available. Individual tests within the battery were not able to predict safety to drive with sufficient accuracy to be used on their own. However, those scoring $>6$ on Road Sign Recognition or $>16$ on Square Matrices Directions were highly likely to be safe on the road.

\section{KEY POINTS}

The Nottingham Assessment for Drivers with Dementia (NADD) is a shortened version of the Dementia Drivers Screening Assessment.

Discriminant equations were developed to predict safety to drive.

The NADD was able to classify participants' on-road driving ability as safe or unsafe with $79 \%$ accuracy.

The NADD was better at identifying safe than unsafe drivers.

Those who are predicted to be unsafe should be referred for on-road assessment.

Cut-off values could not be identified for individual tests with sufficient accuracy to be used to predict safety to drive.

The NADD may be used to assess safety to drive among people who are unable to tolerate longer assessments.

\section{REFERENCES:}

Alzheimer Europe (2014) Restrictions on Freedom. Alzheimer Europe, Luxembourg

Coughlan AK, Hollows SE (1985) The Adult Memory and Information Processing Battery. St James University Hospital, Leeds.

Devos H, Akinwuntan AE, Nieuwboer A, Truijen S, Tant M De Weerdt W (2011) Screening for fitness-to-drive after stroke: a systematic review and meta-analysis. Neurology 76(8): 747- 
56 Folstein MF, Folstein SE, McHugh PR, Fanjiang G (2000) Mini-mental State Examination. Psychological Assessment. Resources Inc, Florida

Lincoln NB, Radford KA, Lee E, Reay AC (2006) The assess - ment of fitness to drive in people with dementia. Int J Geriatr Psychiatry 21(11): 1044-51

Lincoln NB, Taylor JL, Vella K, Bouman WP, Radford KA (2010) A prospective study of cognitive tests to predict per-formance on a standardised road test in people with dementia. Int J Geriatr Psychiatr 25(5): 489-96

Lincoln NB, Radford KA (2012) Nottingham Assessment for Drivers with Dementia. Nottingham University, Nottingham http://tinyurl.com/oac5762 (accessed 6 December 2013) Lincoln NB, Taylor J, Radford KA (2012) Inter-rater reliability of an on-road driving assessment for people with dementia. Clin Rehabil 26(9): 836-9

McKenna P (2009) Rookwood Driving Battery. Pearson Assesment, London

McKenna P, Bell V (2007) Fitness to drive following cerebral pathology: the Rookwood Driving Battery as a tool for pre-dicting on-road driving performance. J Neuropsychol 1(Pt 1): $85-100$

Molnar FJ, Patel A, Marshall SC, Man-Son-Hing M, Wilson KG (2006) Clinical utility of officebased cognitive predictors of fitness to drive in persons with dementia: A systematic review. J Am Geriatr Soc 54(12): 1809-24

Nouri FM, Lincoln NB (1994) The Stroke Drivers Screening Assessment. Nottingham Rehab, Nottingham

Nys H, Raeymaekers P (2013) Rights, autonomy and dignity of people with dementia; Can competence assessment and advance directives help to find the right balance between autonomy and protection? King Baudouin Foundation, Brussels http://tinyurl.com/qcjoebo (accessed May 2014) Reger MA, Welsh RK, Watson GS, Cholerton B, Baker LD, Craft S (2004) The relationship between neuropsychological functioning and driving ability in dementia: a meta-analysis. Neuropsychology 18(1): 85-93

Seiler S, Schmidt H, Lechner A, Benke T, Sanin G et al (2012) Driving Cessation and Dementia: Results of the Prospective Registry on Dementia in Austria (PRODEM). PLoS ONE 7(12): e52710

Unsworth CA, Baker A, Taitz C, Chan SP, Pallant JF, Russell KJ et al (2012) Development of a standardised Occupational Therapy Driver Off Road Assessment (OT-DORA) battery to assess older and/or functionally impaired drivers. Aust Occup Ther J 59(1): 23-36

Vella K, Lincoln NB (2014) Comparison of Assessments of Fitness to Drive for People with Dementia. Neuropsychol Rehabil (in press) 
Warrington EK, James M (1991) The Visual Object and Space Perception Battery. Thames Valley Test Company, Titchfield Wilson BA, Alderman N, Burgess PW, Emslie H, Evans JJ (1996) Behavioural Assessment of the Dysexecutive Syndrome. Thames Valley Test Company, Bury St Edmunds Wilson S and Pinner G (2013) Driving and dementia: a clinician's guide. Adv Psychiatr Treat 19: 89-96 